


\title{
Literatura y memoria como experiencias de paz en la escuela
}

Volumen $6 \mathrm{~N} .{ }^{\circ} 46$ enero - junio de 2019

ISSN: 0122-4328

ISSN-E: 2619-6069

pp. $13-22$

\author{
Literature and \\ Memory as \\ Experiences of Peace \\ in School
}

Literatura e memória

como experiências

de paz na escola

\section{Blanca Nidia Pérez Duarte* \\ Edwin Andrés Toro Rengifo"}

Fecha de recepción: 26-06-18

Fecha de aprobación: 26-09-18

\section{PARA CITAR ESTE ARTÍCULO}

Pérez, B. N. y Toro, E. A. (2019). Literatura y memoria como experiencias de paz en la escuela. Nodos y nudos, 6(46), 13-26. doi: 10.17227/nyn.vol6.num46-8239

\footnotetext{
Licenciada en Ciencias Sociales, especialista en Pedagogía de la Universidad Pedagógica Nacional. Instituto Pedagógico Nacional. blancanidiaicef@yahoo.es

* Licenciado en Español y Literatura de la Universidad Tecnológica de Pereira, especialista en Pedagogía de la Universidad Pedagógica Nacional. Instituto Pedagógico Nacional. 


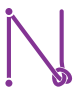

Volumen 6 N. ${ }^{\circ} 46$ enero - junio de 2019 ISSN: 0122-4328 ISSN-E: 2619-6069 pp. $13-22$

\section{RESUMEN}

Este artículo es una reflexión a partir del proceso y la experiencia que significó el proyecto de investigación "La literatura como universo simbólico de la memoria" apoyado por el Centro de Investigaciones de la Universidad Pedagógica Nacional (CIUP), sobre la implementación de una propuesta didáctica de la memoria histórica en la escuela, que parte de la literatura como dispositivo pedagógico. Las preguntas clave que intentaremos responder son ¿por qué hacer memoria?, abordaje que reconoce expresiones particulares y colectivas que se han invisibilizado, fracturando así tejidos sociales fundamentales en la construcción de una sociedad más incluyente, justa y equitativa; ¿por qué desde la literatura?, reconociendo, por un lado, que el conflicto armado incidió en la producción estética y literaria del país, pero también que la literatura es una expresión misma de la memoria que, en términos didácticos, permite el fortalecimiento de la imaginación literaria; y ¿por qué desde la escuela? Este último cuestionamiento se asume con una postura protagonista desde el empoderamiento de sujetos éticos y políticos que resignifican la construcción tanto de la escuela como del país. Las anteriores reflexiones enmarcadas en el contexto del conflicto armado colombiano pretenden, desde el escenario educativo, reconocer tanto a los sujetos como a la escuela en su esencia crítica constructora de tejido social.

Palabras clave: memoria histórica; literatura; proyectos pedagógicos

\section{ABSTRACT}

This article is a reflection from the process and experience that the research project "Literature as a symbolic universe of memory" meant, supported by the Centro de Investigaciones de la Universidad Pedagógica Nacional (CIUP), on the implementation of a didactic proposal of historical memory in school, which starts from literature as a pedagogical device. The key questions that we will try to answer are: why to do memory?, an approach that recognizes particular and collective expressions that have become invisible, thus fracturing fundamental social fabric in the construction of a more inclusive, fair and equitable society; Why from the literature ?, recognizing, on the one hand, that the armed conflict affected the aesthetic and literary production of the country, but also that literature is an expression of memory itself that, in didactic terms, allows the strengthening of the literary imagination; and why from school? This last questioning is assumed with a leading position from the empowerment of ethical and political subjects that resignify the construction of both the school and the country. The previous reflections framed in the context of the Colombian armed conflict aim, from the educational stage, to recognize both the subjects and the school in their critical constructive essence of social fabric.

Keywords: historical memory; literature; pedagogical projects

\section{RESUMO}

Este artigo é uma reflexão do processo e da experiência que o projeto de pesquisa "Literatura como universo simbólico da memória", apoiado pelo Centro de Investigaciones de la Universidad Pedagógica Nacional (cIUP), sobre a implementação de uma proposta didática da memória histórica na escola, que parte da literatura como dispositivo pedagógico. As principais perguntas que tentaremos responder são: por que fazer memória?, uma abordagem que reconhece expressões particulares e coletivas que se tornaram invisiveis, fraturando tecidos sociais fundamentais na construção de uma sociedade mais inclusiva, justa e equitativa; por que da literatura? reconhecendo, por um lado, que o conflito armado afetou a produção estética e literária do país, mas também que a literatura é uma expressão da própria memória que, em termos didáticos, permite o fortalecimento da imaginação literária; e por que da escola? Esse último questionamento é assumido com uma posição de liderança a partir do empoderamento de assuntos éticos e políticos que ressignificam a construção da escola e do pais. As reflexões anteriores enquadradas no contexto do conflito armado colombiano visam, desde o estágio educacional, reconhecer tanto os sujeitos quanto a escola em sua essência construtiva crítica do tecido social.

Palavras-chave: memória histórica; literatura; projetos pedagógicos 


\section{Introducción}

En noviembre de 2015, la comunidad educativa del Instituto Pedagógico Nacional (IPN) asistió por primera vez al Festival Artístico de la Memoria, un evento que rendía un homenaje a las víctimas del conflicto armado colombiano, desde la perspectiva de los mismos estudiantes, que expusieron alli sus narrativas de esa historia reciente. Era un recorrido por imágenes, versos, relatos y videos que recreaban el dolor de un país que, ante todo, queria renacer y reconstruirse. A su vez, este evento era el resultado de una experiencia de investigación-acción participativa que había iniciado un año antes alrededor de la relación memoria/ literatura en la escuela, como experiencias para discutir la paz.

El proyecto "La literatura como universo simbólico de la paz y la memoria en la escuela"1 surgió con el objetivo de diseñar una propuesta curricular para la educación básica y media sobre memoria histórica, tomando como referente una experiencia piloto con docentes del IPN y de la Universidad Pedagógica Nacional (UPN) que vincularan la literatura testimonial o la novela de la violencia a su práctica pedagógica escolar. La memoria histórica se constituía entonces en un campo de saber emergente, que entraba a dialogar con las perspectivas de enseñanza de la Historia y se preguntaba por su relación con áreas del saber como el arte, la literatura o la filosofía.

Con respecto a la Historia, se iniciaba una reflexión que llevaba a una ruptura con la tradición de la trasmisión mecánica de una historia de Colombia de héroes y fechas que desconocen la participación de los sujetos y la afectación social que estos sufren. Y con la reflexión sobre las otras disciplinas, se buscaba que los jóvenes plasmaran preguntas y exploraran lenguajes estéticos que dejaran atrás respuestas cerradas y currículos rígidos que tal vez la escuela ha reproducido tradicionalmente y que no permitía dudas o cuestionamientos, siguiendo así a Freire (citado por Rueda y Quintana, 2004), en su planteamiento "los estudiantes deben ser sujetos de acción, con voz propia,

1 Proyecto financiado con recursos del ciup - Centro de Investigaciones de la Universidad Pedagógica Nacional, en el que participaron en calidad de investigadores: Nylza Offir García Vela y Fernando González Santos (UPN); Blanca Nidia Pérez Duarte y Edwin Toro Rengifo (IPN). antes que depositarios de información". Así entonces, con el trabajo de memoria, los jóvenes tenían la palabra, debían empoderarse de su compromiso histórico con el país, que les exigía un discurso crítico, más allá del reproduccionismo; y otras formas de lenguaje, que se convirtieran en nuevos medios de expresión.

El primer momento de la experiencia fue la indagación por los saberes de los estudiantes con respecto a la historia del conflicto armado en Colombia. En ese sentido, se dio inicio a conversaciones que debian liderar los estudiantes y que arrojaron, en primera medida, una apropiación de la historia desde las cifras, las fechas, los datos, apoyados principalmente en el discurso de los medios de comunicación. Sin embargo, su acercamiento a la dimensión humana del conflicto, a las historias de sus actores, de sus víctimas, era casi inexistente. En ese sentido, la literatura se convirtió en el dispositivo portador de historias que contaban el conflicto desde acontecimientos, personajes y lugares que constituyeron el cuerpo simbólico de la memoria: la voz de los actores armados, de las víctimas, de los muertos, los desaparecidos, las familias; las iglesias, las plazas públicas, las escuelas y las selvas que se convirtieron en los campos de batalla y confrontación; el secuestro, la muerte, la extorsión y, en general, el dolor como resumen de una guerra que resulta menos cruel en la ficción literaria que en la vida misma. El corpus de novelas incluyó: Los ejércitos, de Evelio Rosero; Vivir sin los otros, de Fernando González Santos; El gato y la madeja perdida, de Francisco Montaña; Abraham entre bandidos, de Tomás González, y El olvido que seremos, de Héctor Abad Faciolince.

Fueron meses de trabajo, en los cuales las aulas albergaron, a través de la literatura, el universo de una historia reciente; todavía viva para muchos colombianos. Desde la asignatura de Lengua Castellana, se privilegió el reconocimiento de la novela de la violencia como propuesta estética que dialogaba con el contexto que vivía el país; desde las Ciencias Sociales, se reconocian algunas recurrencias que permitían entender las diferentes formas en que los colombianos vivieron el conflicto armado, comprendiéndolo desde una dimensión amplia que involucrara, a su vez, lo político, económico, social y cultural. Cada docente, 
según el grado que tenía a su cargo, diseñó estrategias que articularan ambas experiencias, la de la literatura y la de la memoria, de tal forma que, a través de ellas, los estudiantes empezaran a pensar la paz, como un estado necesario para el presente y el futuro del país.

En grado octavo, por ejemplo, los estudiantes hicieron énfasis en la construcción de cartografías de la memoria, definidas como representaciones gráficas que dieran cuenta de los personajes, los lugares y las acciones que constituian, de manera estructural, el conflicto armado. Para llegar a esas cartografías, se trabajó con los estudiantes la lectura en voz alta, la creación de museos literarios con los personajes de las novelas y debates orientados por los maestros. En grado noveno, los estudiantes, siguiendo una trayectoria similar, apostaron por el lenguaje del arte para expresar sus reacciones ante los hechos que se contaban en las novelas; surgieron asi el horror, la tristeza, la melancolía y la solidaridad convertidos en obras plásticas, musicales y cinematográficas. En la educación media, grados décimo y undécimo, se privilegió más el pensamiento crítico, en cuanto los estudiantes participaron en debates, conversatorios y crearon escrituras argumentativas que permitieran comprender su visión argumentada del conflicto armado.

Lo anterior da cuenta, aunque a grandes rasgos, de lo que significó la experiencia en el aula de clases: lectura, discusión, contextualización, reflexión y expresión; todo ello, como trayectoria articuladora en los diferentes grados de la institución. Todo, finalmente, se articuló con el Festival Artístico de la Memoria, lo que permitió que el ejercicio de lectura trascendiera el aula y se convirtiera en un acontecimiento pedagógico. Con los estudiantes se llevó a cabo una estrategia de socialización, que aclaró las bases de participación, los tiempos, las modalidades (en las cuales se incluian textos literarios, audiovisuales, musicales u obras de arte) y el requisito único de trabajo: que su producto tuviera como referente al menos una de las novelas del corpus. Era necesaria esta socialización porque el Festival era un concurso, una muestra; pero, para nosotros, era el pretexto para reconstruir el relato de los jóvenes sobre la historia reciente de su país.
Esta "metodología" desmarca la investigación de los métodos pedagógicos que piensan por el maestro; y ratifica la afirmación de un sujeto portador de saberes, capaz de transformar sus propias prácticas. También resignifica una concepción de estudiante como un sujeto que construye su propia ciudadanía activa, participa en las coyunturas de su contexto y aborda de manera crítica los llamados que le hace la escuela para pensarse los lenguajes de expresión. El planteamiento de estas discusiones legitima tanto a los sujetos de la escuela, maestro y estudiantes, ambos poseedores de saberes, como a la construcción de conocimientos que reconozcan el contexto y el sentido de la escuela.

\section{¿Por qué hacer memoria?}

Es casi natural, como lo plantea Ruiz (2013), que "en la escuela se encuentran grupos etarios con vivencias diferenciales y con una significación peculiar de sus experiencias", pero es precisamente en este momento histórico que estas generaciones se encuentran más cercanas en el hecho de querer construir una sociedad que entiende la paz no como un decreto gubernamental, sino como obra colectiva que incluye a todas sus expresiones culturales y no desaparece a ninguno de sus actores. Por el contrario, los enmarca como sujetos con derechos que construyen la riqueza de esta sociedad con proyección hacia la preservación, desde un actuar político con la caracterización de una identidad colectiva, en el espacio escolar en el cual cobra total relevancia el cuidado, la restitución y la creación del tejido social.

Entonces, en el marco de la educación, concebida esta como un "vehículo privilegiado en la democratización de la sociedad" (Carvalho, citado por Jelin, 2004, p. 164), toma relevancia la relación entre los fundamentos teóricos y pedagógicos de la propuesta a través del trabajo de memoria en el cual se recupera a la escuela como eje integrador y de cambio (Jelin, 2004, p. 164), en contextos nacionales que reconocen los sujetos como constructores de comunidades de paz.

¿Quién es víctima? ¿Colombia está en conflicto? ¿La historia constante ha sido el conflicto? ¿Qué versión presentan los medios de comunicación? ¿Somos la generación de la paz? Y finalmente surgió también 
la pregunta por la identidad del docente en el momento actual. Son cuestionamientos que, aunque desprevenidos, evidencian la conciencia histórica que se está instituyendo en los jóvenes. Estos interrogantes han dinamizado durante los últimos años reflexiones en el contexto educativo y han generado intereses no solo por conocer, sino también por comprender procesos políticos, sociales y culturales de la historia de Colombia, de esta manera acercando estos procesos a maestros y estudiantes interesados por encontrar estas respuestas.

Las anteriores preocupaciones invitan a extender el planteamiento ético político realizado por Ortega y Castro (2010) desde la pedagogía de la memoria. Como sujetos, docentes o jóvenes afectados por la violencia política y preocupados por la construcción de una Colombia -fundamentada en la democracia, la solidaridad y la justicia- asumen responsablemente la necesidad del perdón/olvido y el reconocimiento/dignificación de las víctimas del conflicto político.

Como maestros se asume la necesidad de deconstruir los mensajes que llegan de todos los contextos; televisivos, de internet, del comercio, de la música, de la misma escuela; en donde todos ellos imponen una homogeneidad con las ideologías, finalmente una sola por muerte o supresión de las demás. Se precisa, para lo anterior, un maestro como el maestro artesano que cualifica cada vez su quehacer -quehacer pedagógico-, en resignificación de la construcción del sujeto particular y en busca del bienestar general, aun superando las condiciones sociales y económicas del contexto. Sujeto, actor o víctima del conflicto que puede visibilizar, dignificar y restituir su fundamento dentro del tejido social solo desde la escuela con un maestro empoderado en su misión.

$Y$ es en definitiva en este momento crucial del país y de la escuela que emerge la necesidad de paz como construcción colectiva que incluye a todos los sujetos de la misma sociedad y visibiliza las victimas, hasta ahora desconocidas, sujetos que desde la propuesta pedagógica busca no solo entrever, sino también posesionar como un papel trascendental en el proceso histórico de nuestro país.

Así, la postura que se asume en la práctica pedagógica cotidiana "de generar en la ciudadanía aquellos conocimientos que le han de permitir ubicarse en el mundo y participar en su transformación y en su mejora" (Pagès y Santisteban, 2013, p. 15), permite que quede atrás el olvido del papel político de la escuela y así se asuma la formación de sujeto y la transformación social. Se toma entonces; como objetivo de esta propuesta fortalecer esta postura como papel indispensable de la escuela en la construcción no solo una generación, sino toda una sociedad de la paz.

La necesidad desde la visión pedagógica crítica parte de asumir el compromiso social que tenemos los maestros frente a la resignificación de construcción de una sociedad más justa y equitativa que se fundamente en la esencia de lo humano anclado al bienestar tanto particular como colectivo. Este compromiso es aún mayor cuando el quehacer del maestro artesano se proyecta en la formación de niños y jóvenes. La transformación deja atrás el trabajo fundamentado en la repetición de contenidos ajenos a los estudiantes y se acerca a prácticas y procesos evaluativos centrados en los desarrollos particulares tanto de los sujetos como de los contextos y asumir de manera consciente la misión de formación de personalidades y conciencias.

Este eje esencial se establece desde Freire, ya que "la pedagogía consiste, ante todo, en una reflexión acerca de la práctica y del contexto desde unas opciones emancipadoras para favorecer la construcción de un sujeto social protagónico que toma su especificidad de acuerdo con contextos muy definidos y con historias de sus colectividades" (Freire, citado por Ortega, 2009, p. 27). Así, el proyecto se propuso disminuir los espacios lejanos de participación ciudadana que constantemente se enmarcan en dinámicas meramente institucionales -reproduciendo así una exclusión social-, y se quiso despertar la búsqueda de identidad de los jóvenes.

Retomando a Fernández (2001), como toda construcción social, la ciudadanía, y en este caso la participación es enseñada y aprendida desde esta propuesta se invita a la escuela a tener en cuenta los elementos necesarios para esta ciudadanía: los conocimientos, las habilidades, los valores, el entendimiento de la participación como eficaz y relevante, y por último, las oportunidades para la participación efectiva (Sabatini, 
1998) en estudiantes del IPN que reconocen el conflicto como una oportunidad para construir identidad y vínculos sociales.

\section{¿Por qué desde la literatura?}

Una historia de la literatura colombiana contemporánea es una historia del conflicto reciente; es una lectura necesaria sobre la violencia y todas sus formas, puesto que el contexto social, político, económico y cultural de Colombia permeó la narrativa y dio origen a una estética particular sobre la violencia y el conflicto. Por eso, a través de esta experiencia se le apostó a dos perspectivas: por un lado, la formación de lectores críticos capaces de leer el texto literario en contexto, es decir, reconocer la dialéctica entre la forma y contenido, y las condiciones de emergencia que hicieron posible el surgimiento de estas narrativas en la historia reciente del país. Lo anterior, pone de manifiesto la segunda perspectiva, que es asumir la literatura como una forma de hacer memoria y, por consiguiente, contrastar con la visión de otros discursos.

En ese sentido, cobra relevancia la apuesta de convertir a la literatura en el dispositivo pedagógico a través del cual los estudiantes abordarian la temática; la literatura y no el documental, no el informe oficial, no la noticia o la crónica, no el testimonio. La premisa de trabajo es que la literatura es, en sí misma, un relato del conflicto, donde cobran sentido las historias de personajes representados de manera ficcional, pero que guardan relación directa con las historias reales de las víctimas reales.

El potencial que tiene la literatura es que permite desarrollar la imaginación literaria que, en palabras de Martha Nussbaum (1995), puede provocar "un efecto subversivo frente a la idea de racionalidad expresada habitualmente por la ciencia económica utilitarista" porque la literatura "se centra en lo posible, invitando a sus lectores a preguntarse acerca de sí mismos. [...] las obras literarias invitan a sus lectores a ponerse en el lugar de gentes de muy diversos tipos y a asumir sus experiencias" (pp. 42-44). Es decir, en el marco de lo posible, como categoría sustancial de lo literario, lo que le ocurre a un personaje literario le podría ocurrir al lector o a cualquier otro sujeto; y el desarrollo de la lectura da herramientas de vida para ver los desenlaces y formas de actuación. Y esa imaginación literaria no sería posible sin el placer del texto y el placer del reconocimiento que sirven como conducto a través del cual se establece la relación texto/lector.

Ese placer del texto no es necesariamente triunfante, confortante; por el contrario, puede llevar a un estado de caos y desolación, producto de su relato desgarrador. Y para el reconocimiento, no necesariamente se tiene que vivir en un espacio y tiempo similar al del personaje; la simpleza de identificarse en lo humano mueve la empatía y la sensibilidad.

Leer la novela de la violencia implica acercar a los estudiantes a toda una tradición crítica y literaria que inicia con el realismo europeo, pero que encuentra sus formas particulares en el contexto latinoamericano. Novelas que, como se mencionó al inicio del texto, se convierten en el relato del conflicto, en un discurso que, en ocasiones, es el único que encuentran las víctimas o los grupos oprimidos ante el incesante número de publicaciones oficiales que se centran solo en los datos y las cifras.

Por último, es importante mencionar el saber vinculado con la sensibilidad y la imaginación. Esa sensibilidad trasciende el sujeto escolar y se piensa el sujeto ciudadano. Nuevamente, no es la literatura un medio para formar en valores; pero sí la literatura como saber para despertar la sensibilidad humana, la emotividad y la trascendencia de lo real, que son necesarias para la valoración del texto literario. Un conflicto armado que era ajeno para los estudiantes se convirtió en un interés legítimo, desde el reconocimiento y la empatía con las víctimas.

\section{¿Por qué desde la escuela?}

En el marco de la educación en la escuela se plantea modificar el hecho de llevar los estudiantes hacia una escuela-historia tradicional que reproduce los beneficios de una élite, de la Iglesia o de la masificación intencionada del Estado, para integrarlos en la sociedad, y por ello se proyecta como objetivo una educación que permite que los estudiantes construyan su propia personalidad con representaciones simbólicas ajustadas a sus identidades permitiendo prevenir futuros conflictos que los eliminen como sujetos y fundamentándose mejor en la cohesión social que los reconoce y vincula. 


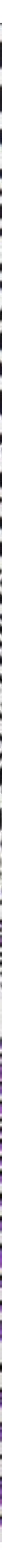

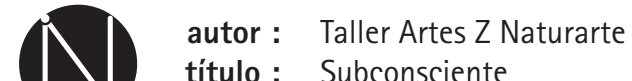 \\ $\begin{aligned} \text { título : } & \text { Subconsciente } \\ \text { año : } & 1995\end{aligned}$}


Dentro del proyecto toma gran relevancia el adoptar como guía orientadora la pregunta, vista desde Gadamer, como horizonte planteado desde un sujeto cambiante; y la configuración política, dejando atrás la simplicidad de la institucionalización para asumirla como un bien-común que permite a los sujetos sentirse vinculados entre sí, sin conflictos excluyentes y por el contrario reconociéndose como ciudadanos de derechos y reconfigurando su propia historia.

Las preguntas que realizan tanto los maestros como los estudiantes demuestran la conciencia para querer dejar atrás historias tradicionales que perpe-

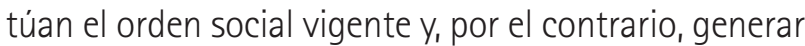
cambios que contengan historias de vida, historias de nuestra historia: ¿Quiénes son las verdaderas víctimas en el conflicto? ¿Por qué en la escuela no se habla de conflicto? ¿Por qué la historia siempre habla de los héroes? ¿Por qué la escuela y la política han invisibilizado el conflicto? Es así como en el marco de la educación en la escuela y retomando a Ortega y Castro (2010), la pedagogía implica el reconocimiento de principios éticos y una apuesta política que permita a los jóvenes prevenir la eliminación de un "otro" y sí fundamente una cohesión social que reconoce y vincula a todos los sujetos de la sociedad.

Pero los anteriores cambios hacia una reconstrucción social en el marco de la globalización "como conjunto de procesos de homogenización y, a la vez, de fraccionamiento articulado del mundo, que reordenan las diferencias y las desigualdades del mundo, sin suprimirlas" (Garcia Canclini, 1999, p. 48); es decir, la necesidad de que la escuela asuma el compromiso histórico de reconocimiento de subjetividades desconocidas o desaparecidas.

Tradicionalmente la escuela y su quehacer pedagógico se han debatido en la dualidad sobre el conocimiento; si este se asume hacia el poder como reproductor de las condiciones existentes, hacia el mantenimiento de un orden social especifico, en el que los sujetos son formados para reconocer y asumir su lugar dentro del funcionamiento de la estructura social, o si por otro lado, es posible pensar en un conocimiento que establezca reflexiones críticas sobre el saber, orientando su acción hacia la contribución de una sociedad más justa y equitativa. Si se asume la primera visión, los sujetos educativos no tienen mayores posibilidades de transformar o incidir en el mundo de la escuela y menos aún en el conjunto de la sociedad, pues su rol está predeterminado por la naturaleza de la institución escolar y del conocimiento que ejercen, mientras que desde la segunda perspectiva, los sujetos son partícipes decisorios en el proceso, las elecciones que toman tienen incidencia directa en el rumbo de la escuela, sus posicionamientos particulares pueden transformar el funcionamiento de esta, basada en la constitución de sujetos y sociedades autónomos.

La anterior visión permite que desde el desarrollo del proyecto los sujetos se asumen no como meras herramientas al servicio de una verdad externa a ellos, ya sea esta entendida como una verdad reguladora o emancipadora, incluso el reposicionamiento es aún más profundo, pues permite entender la verdad, o el saber, como sometido a las necesidades del sujeto, invirtiendo la relación establecida tradicionalmente, en la cual era el sujeto el que debía moldearse acorde al discurso que lo define. Por el contrario, es la posibilidad de comunicación, de ponerse en juego frente al otro, lo que cobra un valor especial en esta propuesta, asi las opiniones, los prejuicios, las historias personales, las fallas y los fracasos, no se entienden como un elemento que debe ser omitido de la relación intersubjetiva, sino como aspectos fundamentales para poder establecer procesos de comunicación reales y transformadores entre sujetos. Ahora bien, desde el objetivo de esta propuesta es pertinente además de buscar la transformación "reconstruir atmósferas [...] ya que implica captar la mentalidad colectiva, las dimensiones de la cotidianidad, los alcances de los mensajes ideológicos y políticos" (Philippe Joutard, citado por Jiménez, 2004, p. 148)

Transformación desde la experiencia, las inquietudes, los intereses tanto de los estudiantes como de los maestros, y como docentes se debe visualizar la criticidad frente a las presiones que directa o indirectamente recibe el proceso educativo, desde la reproducción del Estado desde adentro, o desde afuera con los procesos de globalización. Se debe tratar de estudiar para someter a crítica profunda todo lo nuevo y articularlo con lo cotidiano a un nuevo conjunto de contenidos y métodos adaptados a la situación local. 
Es decir, un pais de tensiones entre las permanencias culturales y las presiones hacia el cambio.

\section{Conclusiones}

Los estudiantes lograron trayectorias sensibles hacia una relación empática con las víctimas del conflicto, a partir de los personajes presentes en las novelas. Sus relatos anteriores a esta experiencia (saberes previos) configuraban un discurso a partir de sujetos colectivos, deshumanizados y sobre todo lejanos para ellos en el espacio y el tiempo. Liberales, conservadores, guerrilleros, paramilitares... la lista es larga, pero no existía, ni siquiera como nominación sustantiva genérica, la víctima. Las lecturas literarias los llevaron a nuevas narrativas sobre el conflicto, donde las víctimas, con nombre o drama humano particular, eran el centro. Todo esto se pudo evidenciar en las cartografias, las piezas artísticas, musicales y cinematográficas, en los ensayos y en sus discusiones de clase.

Por otro lado, esta experiencia dejó la posibilidad de que, tanto estudiantes como maestros, se reconocieran como víctimas indirectas del conflicto, frente a un Estado que, ante la urgencia de acabar el conflicto, soslaya otras problemáticas sociales que afectan a todos. Lo anterior marca una nueva perspectiva en la concepción del sujeto frente a su formación crítica y política.

Es dialéctica la manera de asumir el proyecto por parte de los maestros a los cuales se les invita a participar del mismo, ya que maestros de las asignaturas mencionadas que deciden participar activamente en la propuesta manifiestan el proceso trasversal que se aborda -memoria, conflicto y literatura- e invitan a seguir fortaleciendo el trabajar por proyectos interdisciplinares; primero porque permite a los mismos maestros plantear estrategias en equipo, segundo y principalmente porque permite a los estudiantes aprovechar y relacionar todas las apuestas didácticas en el sentido de apropiarse y comprender los procesos trabajados pedagógicamente.

Durante el planteamiento espontáneo del proyecto a maestros de las diferentes asignaturas del IPN se percibió una alta sensibilidad por la problemática a trabajar como lo era el conflicto y la memoria desde un marco literario, "es interesante trabajarlo no desde lo que dice el gobierno o la historia tradicional" decía alguno de ellos. Sin embargo, al proseguir hacia el planteamiento de estrategias propuestas por ellos mismos para el trabajo curricular, se evidenció con mayor ímpetu la inquietud por cumplir con objetivos ya establecidos por los mismos maestros o por las mismas áreas, evidenciando así las pocas flexibilidades que pueden tener algunos currículos, los temores por asumir una real autonomía institucional o los inconscientes temores por el manejo del tiempo o el control disciplinario.

\section{Referencias}

Fernández, G. (2001). La ciudadanía en el marco de las políticas educativas. Revista Iberoamericana de Educación, 26, 167-199. https://doi.org/https://doi.org/10.35362/ rie260983

García Canclini, N. (1999). Globalización imaginaria. Buenos Aires, Argentina: Paidós.

Jiménez. A (2004). Algunos elementos para la investigación en historia. En A. Torres Carrillo (comp.), La práctica investigativa en ciencias sociales (pp. 136-152). Bogotá: Universidad Pedagógica Nacional.

Jelin, E. (2004). Educación y memoria: la escuela elabora el pasado. Madrid: Siglo Xx। Editores.

Nussbaum, M. (1995). La imaginación literaria en la vida pública. Isegoria, 11, 42-80.

Ortega, P. (2009). La pedagogía crítica: reflexiones en torno a sus prácticas y sus desafíos. Pedagogía y Saberes, 31, 26-33.

Ortega, P. y Castro, C.P. (2010). Rostros y rastros de una pedagogía de la memoria. Nodos y Nudos,3(28), 81-91.

Pagès, J y Santisteban, A. (2013). Una mirada al pasado y un proyecto de futuro. Investigación e innovación en didáctica de las ciencias sociales. España: Red Iberoamericana de Didáctica de las Ciencias Sociales.

Rueda, R. y Quintana, A. (2004). Ellos vienen con el chip incorporado. Aproximación a la cultura informática escolar. Bogotá: IDEP.

Ruiz, A. (2013). ¿Qué contiene una escuela? Sobre la dimensión política de la convivencia escolar. Bogotá, Colombia: Universidad Pedagógica Nacional (UPN).

Sabatini, F. (1998). Direcciones para el futuro. R. Jordán y D. Simioni (comps.), Ciudades intermedias en América Latina y el Caribe: Propuestas para la gestión urbana (pp. 127-214. Santiago: CEPAL/Ministero degli Affaire Esteri Cooperazione Italiana. 


\section{Diálogo del conocimiento}

El proyecto Literatura y memorias como experiencia de paz en la escuela es una invitación para reconstruir hechos y acontecimientos que determinan la historia de Colombia y que en el contexto escolar se convierten en lecciones de vida. A pesar del dolor que ha producido la violencia en nuestro país, en muchos casos incomprensible y aterradora, y los riesgos que implica abordar estos asuntos en el aula de clase, los maestros del Instituto Pedagógico Nacional asumen el proyecto como una oportunidad para develar la importancia del trabajo pedagógico colaborativo, que propicia otras alternativas para indagar, reflexionar, comprender, enseñar y aprender acerca de un hecho histórico, a través de diálogos, narrativas, expresiones artísticas, ejercicios de investigación, entre otros.

El proyecto es una apuesta por la pedagogía y por la reivindicación de otras maneras de asumir el quehacer escolar, a partir de la riqueza que emerge de la sensibilidad y de la convicción de que el espacio propicio para leer, indagar, y comprender la realidad social es la escuela. Es un llamado de atención para reconstruir prácticas que trasciendan el libreto de textos o guias estandarizadas. Se destaca de este proyecto que los conflictos sociales que se develan en la escuela no se abordan únicamente con conceptos académicos; se comprenden con las prácticas, las experiencias y con las lecciones aprendidas desde expresiones y procesos como los relatos, el arte y la investigación.

La invitación es a recorrer cada párrafo del texto Literatura y memorias como experiencia de paz en la escuela, con la certeza de que en alguna frase o párrafo se encontrará una lección de maestros que inspiran, que dejan huella y para quienes abordar temas como el conflicto armado colombiano en sus aulas de clase es parte del compromiso social de educar. Por lo tanto, sus lecciones serán siempre un legado que invita a la reflexión, a la participación activa y consciente, a la construcción, a la concertación, a la movilización y al cambio. Lo contrario es la indiferencia, el anquilosamiento, el silencio como fruto de la intimidación y el miedo, en otras palabras: la resignación.

Pilar Rubio

Maestria en Supervisión Educativa Universidad de Antioquia 